\title{
Neural Processing of Calories in Brain Reward Areas Can be Modulated by Reward Sensitivity
}

\author{
Inge van Rijn ${ }^{1 *}$, Sanne Griffioen-Roose ${ }^{1}$, Cees de Graaf ${ }^{1}$ and Paul A. M. Smeets ${ }^{1,2}$ \\ ${ }^{1}$ Division of Human Nutrition, Wageningen University and Research Centre, Wageningen, Netherlands, ${ }^{2}$ Image Sciences \\ Institute, University Medical Center Utrecht, Utrecht, Netherlands
}

OPEN ACCESS

Edited by:

Angela Roberts,

University of Cambridge, UK

Reviewed by:

Ivan E. De Araujo,

The John B. Pierce Laboratory, USA Hisham Ziauddeen, University of Cambridge, UK

*Correspondence: Inge van Rijn inge.vanrijn@wur.nl

Received: 06 October 2015 Accepted: 24 December 2015 Published: 14 January 2016

Citation:

van Rijn I, Griffioen-Roose S, de Graaf $C$ and Smeets PAM (2016) Neural Processing of Calories in Brain Reward Areas Can be Modulated by Reward Sensitivity.

Front. Behav. Neurosci. 9:371. doi: 10.3389/fnbeh.2015.00371
A food's reward value is dependent on its caloric content. Furthermore, a food's acute reward value also depends on hunger state. The drive to obtain rewards (reward sensitivity), however, differs between individuals. Here, we assessed the association between brain responses to calories in the mouth and trait reward sensitivity in different hunger states. Firstly, we assessed this in data from a functional neuroimaging study (van Rijn et al., 2015), in which participants ( $n=30$ ) tasted simple solutions of a non-caloric sweetener with or without a non-sweet carbohydrate (maltodextrin) during hunger and satiety. Secondly, we expanded these analyses to regular drinks by assessing the same relationship in data from a study in which soft drinks sweetened with either sucrose or a non-caloric sweetener were administered during hunger $(n=18)$ (Griffioen-Roose et al., 2013). First, taste activation by the non-caloric solution/soft drink was subtracted from that by the caloric solution/soft drink to eliminate sweetness effects and retain activation induced by calories. Subsequently, this difference in taste activation was correlated with reward sensitivity as measured with the BAS drive subscale of the Behavioral Activation System (BAS) questionnaire. When participants were hungry and tasted calories from the simple solution, brain activation in the right ventral striatum (caudate), right amygdala and anterior cingulate cortex (bilaterally) correlated negatively with BAS drive scores. In contrast, when participants were satiated, taste responses correlated positively with BAS drive scores in the left caudate. These results were not replicated for soft drinks. Thus, neural responses to oral calories from maltodextrin were modulated by reward sensitivity in reward-related brain areas. This was not the case for sucrose. This may be due to the direct detection of maltodextrin, but not sucrose in the oral cavity. Also, in a familiar beverage, detection of calories per se may be overruled by a conditioned response to its flavor. In conclusion, the brain reward response to calories from a long chain starch sugar (maltodextrin) varies with trait reward sensitivity. The absence of this effect in a familiar beverage warrants further research into its relevance for real life ingestive behavior.

Keywords: brain reward circuitry, calories, maltodextrin, reward sensitivity, sucrose, taste

\section{INTRODUCTION}

In our Western society, there is an abundance of food cues and an enormous supply of different kinds of appetizing and calorie-rich foods. Therefore, many of us easily engage in overeating. Consequently, it is no surprise that obesity rates are high and still increasing (Ng et al., 2014). However, it is still unclear why some of us are more inclined to engage in overeating than others. 
The answer may lie in how sensitive we are to the food rewards surrounding us. Reward sensitivity is a personality trait that can be described as "the ability to derive pleasure or reward from natural reinforcers like food, and from pharmacological rewards like addictive drugs" (Davis et al., 2004). Reward sensitivity can be measured with the Behavioral Inhibition System and Behavioral Activation System (BIS/BAS) questionnaire (Carver and White, 1994). This questionnaire is based on the theory of Gray (Gray, 1990; Carver and White, 1994; Gray and Mcnaughton, 2003), which describes two neurobiological systems that both respond to environmental cues: the Behavioral Inhibition System (BIS) and the Behavioral Approach System (BAS). The BIS is sensitive to signals of punishment, and activation of this system inhibits behavior and induces negative feelings. The BAS is sensitive to signals of reward and activation of this system promotes behavior and positive feelings. Food reward is reflected by the BAS (Carver and White, 1994). More specifically, the BAS is activated by cues that indicate the possibility of attaining food rewards rather than by food consumption (Corr et al., 2013). Sensory signals like taste and sight of food can be seen as such cues, because they signal the presence of nutrients.

High reward sensitivity has been associated with food cravings, overeating, overweight, obesity and eating disorders (Davis et al., 2004, 2007; Franken and Muris, 2005; Bijttebier et al., 2009; Harrison et al., 2010; Verbeken et al., 2012). Beaver et al. (2006) showed that trait reward sensitivity as measured with the BAS scale, is associated with differential processing of food cues in the brain. In their study, reward sensitivity scores of healthy participants correlated strongly with brain activation by pictures of appetizing foods in reward areas such as the ventral striatum, amygdala, midbrain, and orbitofrontal cortex.

Foods are not only rewarding because of their palatability, but also because of their caloric value. Several recent studies found that oral exposure to calories, independent of sweet taste, induced responses in classical reward areas such as the striatum, anterior cingulate cortex (ACC), and amygdala (Frank et al., 2008; Chambers et al., 2009; Smeets et al., 2011) The presence of calories in the oral cavity may directly signal the imminent arrival of a rewarding (caloric) food. Therefore, it is plausible that neural processing of oral calories may be modulated by reward sensitivity in a similar way as was found for food pictures by Beaver et al. (2006). In addition, several studies found that hunger state interacts with brain activation in response to oral calories (Smeets et al., 2011; van Rijn et al., 2015). Currently, though, it is still unknown in how far reward sensitivity differentially affects brain responses to calories during hunger and satiety.

Based on the above, we hypothesized that (1) brain activation in reward areas in response to oral calories depends on trait reward sensitivity, in particular in the striatum, amygdala and ACC, and (2) that this association will be most prominent during hunger. Thus, we aimed to assess the correlation between reward sensitivity and the brain responses to calories in the mouth in different hunger states. Firstly, we assessed this in data from a functional neuroimaging study (van Rijn et al., 2015), in which simple solutions of a non-caloric sweetener with or without a non-sweet carbohydrate (maltodextrin) were administered during hunger and satiety (van Rijn et al., 2015). Secondly, we sought to extrapolate these findings to regular drinks by assessing the same relationship in data from a study in which soft drinks sweetened with either sucrose or a noncaloric sweetener were administered during hunger (GriffioenRoose et al., 2013). BAS drive and BAS reward, two subscales of the BIS/BAS questionnaire that respectively reflect the tendency to take action in response to a food reward and the amount of positive feelings experienced in response to this reward (Carver and White, 1994; Gomez et al., 2005), were used as measures of reward sensitivity.

\section{MATERIALS AND METHODS}

Data from two separate studies were used. Relevant details are described below. For full experimental details see van Rijn et al. (2015) and Griffioen-Roose et al. (2013).

\section{Participants}

For both studies we recruited healthy, normal-weight (BMI between 18.5 and $25 \mathrm{~kg} / \mathrm{m}^{2}$ ) participants (age between 18 and 35 years). Exclusion criteria were among others: a restrained eating score higher than 2.80 (women) or 2.25 (men) (Dutch Eating Behavior Questionnaire (van Strien, 2005), an energy restricted diet during the past 2 months, change in body weight of more than $5 \mathrm{~kg}$ during the past 2 months, lack of appetite, stomach or bowel diseases, diabetes, thyroid disease or any other endocrine disorder, use of daily medication other than oral contraceptives, having difficulties with swallowing and/or eating, having taste or smell disorders, being allergic and/or intolerant for products under study, smoking more than one cigarette/cigar a day, exclusive consumption or avoidance of light versions of beverages, being pregnant or lactating or having any contra-indication for MRI scanning. Thirty female participants completed Study 1 and 18 participants completed the fMRI part of Study 2 (15 men, 3 women, see Table 1). Before enrollment, participants were screened on inclusion and exclusion criteria via a questionnaire including a medical history questionnaire and completed an fMRI training session in which they were familiarized with the fMRI procedure. All participants gave written informed consent. Both studies were conducted according to the principles of the Declaration of Helsinki, approved by the Medical Ethical Committee of Wageningen University and registered in the Dutch Trial Register (Study 1: NTR 3749, Study 2: NTR: 3289).

\section{Study Design}

Study 1 had a randomized crossover design in which participants were scanned on two occasions, once during hunger and once during satiety. During the two scan sessions participants tasted fixed amounts of a control stimulus (water) and five stimuli containing carbohydrates, artificial sweeteners or both (sucralose, maltodextrin, maltodextrin + sucralose, glucose, and fructose solutions), while their brain responses were measured using functional MRI. Here, we focus on the responses to two of these 
TABLE 1 | Participant characteristics.

\begin{tabular}{|c|c|c|}
\hline Characteristics & Study 1 & Study 2 \\
\hline$N$ & 30 & 18 \\
\hline Gender & Female & Male (15) and Female (3) \\
\hline BMl $\left(\mathrm{kg} / \mathrm{m}^{2}\right)^{\mathrm{a}}$ & $22.6 \pm 1.4$ & $22.1 \pm 1.6$ \\
\hline Age $(\text { years })^{a}$ & $22 \pm 3$ & $22 \pm 2$ \\
\hline BAS drive score ${ }^{a}$ & $11 \pm 2$ & $12 \pm 2$ \\
\hline BAS drive range & $8-16$ & $9-15$ \\
\hline BAS reward score ${ }^{a}$ & $17 \pm 1$ & $18 \pm 1$ \\
\hline BAS reward range & $15-20$ & $15-20$ \\
\hline
\end{tabular}

${ }^{a}$ Mean $\pm S D$.

stimuli, the sweet caloric (maltodextrin + sucralose) and the sweet non-caloric (sucralose) solution.

Study 2 had a randomized crossover design consisting of two periods, which consisted of three parts: a premeasurement, a conditioning period, and a post-measurement. In the conditioning period, subjects were offered a non-caloric sweetened and sugar sweetened version of a soft drink or a yoghurt drink for breakfast (10 times per drink). During scan sessions in the pre-measurement and post-measurement periods, participants tasted fixed amounts of the non-caloric sweetened and sugar sweetened drinks and a control stimulus (water) while their brain responses were measured using functional MRI. Here, we further analyze the brain responses to tasting the non-caloric sweetened and sugar sweetened soft drinks in the pre-measurement period.

\section{Stimuli}

The sweet non-caloric solution and the sweet caloric solution, used in Study 1, were made by dissolving, sucralose (Brenntag specialties, $0.254 \mathrm{~g}$ SPLENDA ${ }^{\circledR}$ Sucralose per liter, $0 \mathrm{~kJ} / 0 \mathrm{kcal}$ per liter) and maltodextrin + sucralose $(158.2 \mathrm{~g}$ Nutricia Fantomalt (90\% polysaccharides - DE 19, 6\% mono/disaccharides) $+0.140 \mathrm{~g}$ SPLENDA ${ }^{\circledR}$ Sucralose per liter, $2541 \mathrm{~kJ} / 607 \mathrm{kcal}$ per liter) in demineralized water. The solutions were equisweet. Sweetness was matched in a pilot study using the method of constant stimuli $(n=10)$. Furthermore, prior to the study, stimuli were rated on sweetness by a trained sensory panel and during the study by the participants. In both cases, no significant differences in sweetness were found between the two solutions (for more details see, van Rijn et al., 2015).

The non-caloric sweetened and sugar sweetened soft drinks used in Study 2 were developed and prepared by Royal Friesland Campina (Amersfoort, The Netherlands) and contained $0 \mathrm{~kJ} / 0 \mathrm{kcal}$ per liter $(0.11 \mathrm{~g}$ sucralose per liter) and $1673 \mathrm{~kJ} / 400$ $\mathrm{kcal}$ per liter $(68.6 \mathrm{~g}$ sucrose per liter). The soft drinks were grape/lemon flavored and matched on sensory characteristics, including sweetness.

\section{BAS Scores}

Reward sensitivity was measured with the Dutch version of the BIS/BAS questionnaire developed by Carver and White (1994). The Dutch BIS/BAS questionnaire was validated by Franken et al. (2005), and is considered a reliable and valid measure. The BAS scale consist of three subscales: BAS drive, BAS reward and BAS fun. BAS drive and BAS reward are most relevant for appetitive motivation and discussed in this paper. "BAS fun reflects the tendency to seek out and impulsively engage in potentially rewarding activities" (Gomez et al., 2005). This scale is not discussed because the food-context of this paper concerns primary reward rather than "activities." Moreover, we investigate a classic well-known reward (food/calories) rather than a potential reward. In addition, BIS scores are also outside the scope of this paper.

The BIS/BAS questionnaire consists of 20 questions. The BAS drive scale is comprised of four of those questions (min-max score: 4-16) and the BAS reward scale of five (min-max score: 5-20). BAS scores for Study 1 were acquired during the fMRI training session and BAS scores for Study 2 were acquired on the last scan day (after scanning). Scores and ranges of BAS drive and BAS reward for Study 1 and Study 2 can be found in Table 1.

\section{Experimental Procedures Study 1}

Participants arrived between 10:25 and 14:00 h at the test location (Hospital Gelderse Vallei, Ede, The Netherlands) after a fast of at least $3 \mathrm{~h}$ (no food, only water). Participants were instructed to eat a small self-chosen breakfast, prior to the $3 \mathrm{~h}$ fast. Hereafter participants were placed in the MRI scanner and scanned while tasting the solutions several times. During the satiety session participants started with an ad libitum lunch consisting of bread rolls $(1063 \mathrm{~kJ} / 254 \mathrm{kcal}$ per $100 \mathrm{~g}$ ), full fat cheese $(1570 \mathrm{~kJ} / 375 \mathrm{kcal}$ per $100 \mathrm{~g})$, boiled eggs $(645 \mathrm{~kJ} / 154 \mathrm{kcal}$ per $100 \mathrm{~g})$, butter $(1549 / 370 \mathrm{kcal}$ per $100 \mathrm{~g})$, sandwichspread $(984 \mathrm{~kJ} / 235 \mathrm{kcal}$ per $100 \mathrm{~g})$, cucumber, tomato, orange juice $(167 \mathrm{~kJ} / 40 \mathrm{kcal}$ per $100 \mathrm{~g})$ and skimmed milk $(197 \mathrm{~kJ} / 47 \mathrm{kcal}$ per $100 \mathrm{~g})$. Participants were instructed to eat until comfortably full. After lunch, the same procedures were followed as during the hunger session.

\section{Study 2}

Participants arrived between 7.00 and $11.00 \mathrm{~h}$ at the study location (Hospital Gelderse Vallei, Ede, The Netherlands) after a fast of at least $3 \mathrm{~h}$ (no food, only water) and were scanned while tasting the soft drinks several times. Note that in this study there was no satiety session.

\section{Scanning Procedure}

In study 1 , a scan session consisted of a high-resolution $\mathrm{T}_{1}$ weighted anatomical scan and 3 functional runs during which 300 functional volumes were acquired using a $\mathrm{T}_{2}^{*}$-weighted gradient echoplanar imaging sequence on a 3-T Siemens Magnetom Verio (Siemens, Erlangen, Germany). During each functional run all solutions were tasted four times, resulting in a total of 12 taste trials per solution per scan session. Solutions were offered in $2 \mathrm{~mL}$ sips in a semi-random order. Each taste event (11 s) was followed by a 3-s swallow, a 4-s rinse with water, a 3-s swallow and a 3- to 5-s rest.

In study 2, a scan session consisted of a high-resolution $\mathrm{T}_{1}$-weighted anatomical scan and 3 functional runs during 
TABLE 2 | Pearson correlation coefficients ( $r$ ) for the correlations between the difference in liking between the caloric and non-caloric stimulus, BAS drive and BAS reward scores.

\begin{tabular}{llll}
\hline Study & $\begin{array}{l}\text { Liking and } \\
\text { BAS drive }\end{array}$ & $\begin{array}{l}\text { Liking and } \\
\text { BAS reward }\end{array}$ & $\begin{array}{l}\text { BAS drive and } \\
\text { BAS reward }\end{array}$ \\
\hline 1 & H: 0.28 & H: 0.05 & $0.38^{*}$ \\
S: -0.08 & S: -0.13 & \\
\hline 2 & -0.03 & -0.04 & 0.28 \\
\hline
\end{tabular}

* Significant at the 0.05 level; H, hunger; S, satiety.

which 262 functional volumes were acquired using a $\mathrm{T}_{2}^{*}$ weighted gradient echo imaging sequence on a 3-Tesla Siemens Magnetom Verio (Siemens, Erlangen, Germany). Each functional run consisted of 5 taste trials for every drink, leading to a total of 15 taste trials per drink. Drinks were offered in $2 \mathrm{~mL}$ sips in a semi-random order. Participants tasted every drink for $11 \mathrm{~s}$ while a picture of the drink was shown, followed by a 3-s swallow, a 4-s rinse with water, a 3-s swallow and a 3 to 5 -s rest.

For both Study 1 and 2, participants rated liking once for every stimulus on a 9-point scale during each functional run. Instructions to either taste, swallow, rate, rinse, or rest were given to participants via visual cues on a screen placed in the bore at the back end of the scanner. Stimuli were administered with the use of programmable syringe pumps (New Era Pump Systems Inc., Wantagh, NY) at $50 \mathrm{~mL} / \mathrm{min}$.

\section{Analysis}

In both Study 1 and 2, functional volumes of every participant were preprocessed and analyzed with the SPM8 software package (Wellcome Department of Imaging Neuroscience, London, UK) in conjunction with the MarsBar toolbox (http://marsbar. sourceforge.net/) run with MATLAB 7.12 (The Mathworks Inc, Natick, MA). Details about the preprocessing steps can be found in van Rijn et al. (2015) and Griffioen-Roose et al. (2013).

In the subject level analyses of Study 1, nine conditions were modeled: delivery of sucralose, maltodextrin, maltodextrin + sucralose, glucose, fructose and water, and swallowing, rinsing and stimulus rating. In the subject level analyses of Study 2, seven conditions were modeled: delivery of the non-caloric sweetened soft drink, sugar sweetened soft drink, tomato juice and water, and swallowing, rinsing and stimulus rating. Responses to swallowing, rinsing, stimulus rating, maltodextrin, glucose, fructose, tomato juice and water are not of interest for answering our current research question and are therefore disregarded. After modeling of the conditions, a so-called contrast image was calculated for every participant by subtracting activation by sucralose from activation by maltodextrin + sucralose (Study 1) or activation by the non-caloric sweetened soft drink from that by the sugar sweetened soft drink (Study 2). For Study 1 , this was done for both the hunger and satiety condition. Subsequently, these contrast images were entered into separate one-sample $t$-tests with liking, BAS reward and BAS drive as covariates (for Study 1 this was done separate for the hunger and satiety condition). Liking was added as a covariate of no interest to regress out possible effects of differences in liking between the stimuli. Using the other two covariates we tested for correlations between BAS drive/BAS reward scores and taste activation across the whole brain. The resulting correlation $\mathrm{T}$ maps were thresholded at $P<0.001$ (uncorrected for multiple comparisons) and a cluster size of $k>9$ contiguous voxels. A priori regions of interest (ROIs) were the amygdala, striatum and ACC. A mask of these regions was created with the WFU Pickatlas tool (Maldjian et al., 2003) and was used to do a ROIanalysis in with small volume correction over the mask volume. Whole brain results are reported in Supplementary Tables 1-3.

\section{RESULTS}

\section{Main Effects}

Main effects for study 1 have been reported in van Rijn et al. (2015). There were no differences in taste activation between the maltodextrin + sucrose and sucralose solution. Main effects for study 2 have been reported in Griffioen-Roose et al. (2013). More activation was found for the sugar sweetened soft drink than for the non-caloric sweetened soft drink in the middle cingulum, precentral gyrus and rolandic operculum.

\section{Correlations between Covariates}

Pearson correlation coefficients for correlations between the covariates used in the analyses (liking, BAS drive and BAS reward) for Study 1 and 2 can be found in Table 2. BAS drive and BAS reward scores obtained during Study 1 correlated significantly $(r=0.38, P<0.05)$.

\section{Study 1: Sugar Solution and Reward Sensitivity}

The ROIs in which correlations between BAS drive scores and brain activation in response to calories (maltodextrin and sucralose minus sucralose) during hunger and satiety were found, are shown in Table 3. BAS drive scores correlated with taste activation in the amygdala, ACC and striatum. BAS reward scores did not correlate with taste activation in any of the ROIs.

Taste activation in the right caudate (ventral striatum) correlated negatively with BAS drive scores during hunger $(r=-0.62$; Figure 1). During satiety, however, BAS drive scores were positively correlated with activation in the left caudate $(r=0.60$; Figure 2). Taste activation in the ACC (bilaterally) and the right amygdala correlated negatively with BAS drive scores during hunger (left ACC: $r=-0.63$, right ACC: $r=-0.59$, right amygdala: $r=-0.48$ ), but not during satiety (Figures 3, 4).

\section{Study 2: Soft Drink and Reward Sensitivity}

Brain activation during tasting of soft drinks with vs. without calories did not correlate with BAS drive and BAS reward scores in any of the ROIs.

\section{DISCUSSION}

We assessed the correlation between reward sensitivity and brain responses to calories in the mouth in different hunger states. Firstly, we assessed this in data from a functional neuroimaging 
TABLE 3 | ROIs in which brain activation by oral calories (maltodextrin and sucralose minus sucralose) correlated significantly with reward sensitivity (BAS drive score) during hunger and satiety.

\begin{tabular}{|c|c|c|c|c|c|c|}
\hline Contrast & Region & Cluster size & Z-score & \multicolumn{3}{|c|}{ Peak coordinates } \\
\hline Positive correlation & No regions were found & & & & & \\
\hline \multirow[t]{5}{*}{ Negative correlation } & R caudate & 54 & 4.15 & 12 & 17 & -8 \\
\hline & R putamen & & 4.09 & 21 & 17 & -8 \\
\hline & R amygdala & 21 & 3.85 & 18 & -1 & -17 \\
\hline & $\mathrm{R}$ anterior cingulate & 74 & 3.72 & 3 & 32 & 16 \\
\hline & $\mathrm{L}$ anterior cingulate & & 3.33 & 0 & 23 & 22 \\
\hline \multicolumn{7}{|l|}{ SATIETY } \\
\hline Positive correlation & L caudate & 15 & 3.76 & -12 & 26 & 4 \\
\hline Negative correlation & No regions were found & & & & & \\
\hline
\end{tabular}
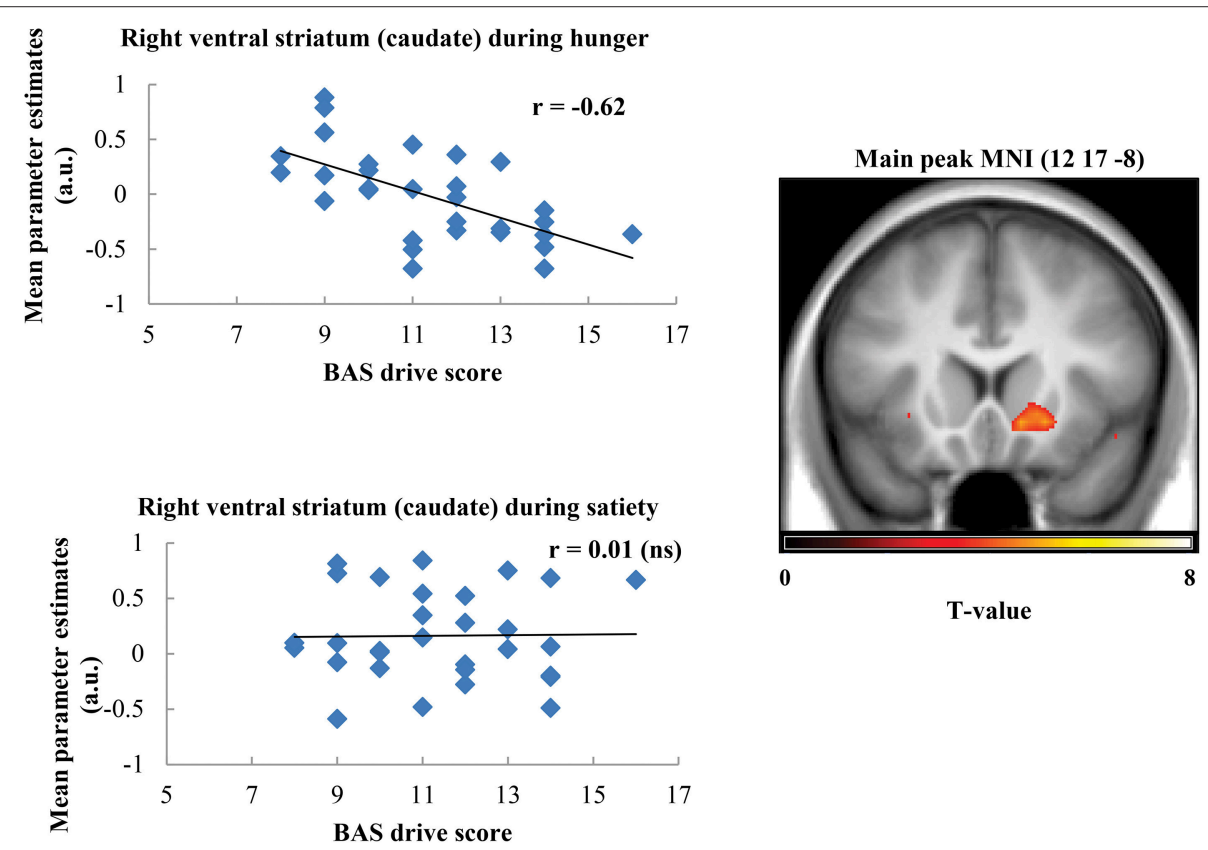

score) during hunger (significant) and satiety (not significant) in the right ventral striatum (caudate).

study, in which simple solutions of a non-caloric sweetener with or without maltodextrin were administered during hunger and satiety (van Rijn et al., 2015). We found that when participants were hungry and tasted calories, brain activation in the right ventral striatum (caudate), amygdala and ACC (bilaterally) correlated negatively with BAS drive scores. In contrast, when participants were satiated, brain responses correlated positively with BAS drive scores in the left caudate. BAS reward scores did not correlated with taste activation in reward related areas.

Secondly, we sought to extrapolate these findings to regular drinks by assessing the relationship between brain responses to calories in the mouth and reward sensitivity in data from a study in which soft drinks sweetened with either sucrose or a noncaloric sweetener were administered during hunger (GriffioenRoose et al., 2013). Here, we found no correlations between reward sensitivity and brain responses to calories in any reward related area.

For simple solutions, correlations with taste activation were found for BAS drive but not for BAS reward. The lack of findings for BAS reward may be explained by the valence of the solutions. BAS reward is related to the degree of positive feelings people experience in response to a reward. Solutions were, on average, disliked by the participants (mean liking scores on 9point scale: 2.9 (maltodextrin + sucralose), 3.4 (sucralose), see 
Left caudate during hunger

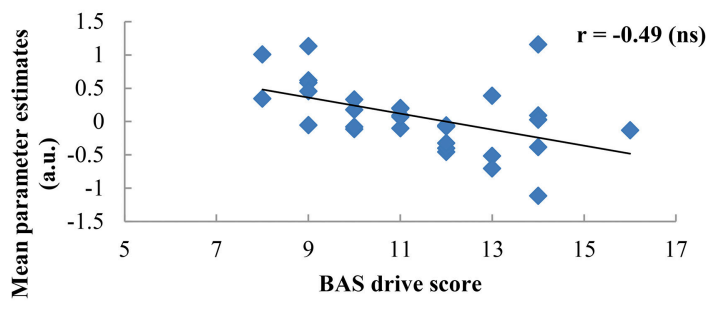

Left caudate during satiety

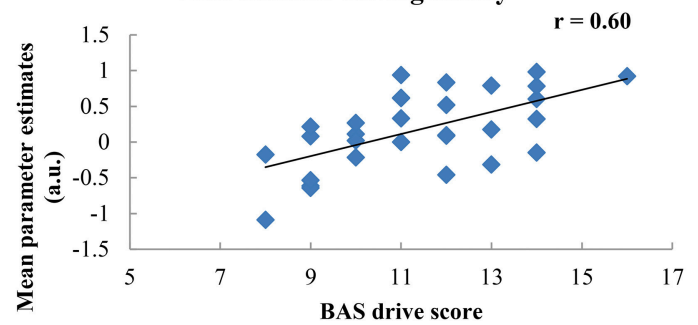

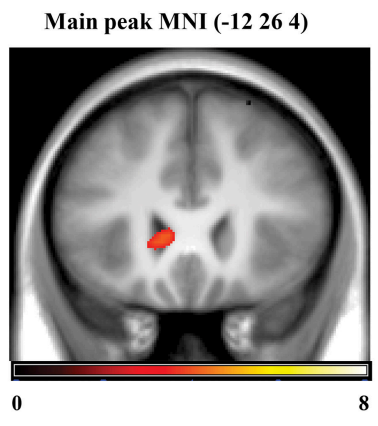

T-value

FIGURE 2 | Scatterplot of brain activation in response to oral calories (maltodextrin and sucralose minus sucralose) and reward sensitivity (BAS drive score) during hunger (not significant) and satiety (significant) in the left caudate.

van Rijn et al., 2015) and probably did not elicit positive feelings. BAS drive scores, which are related to the tendency to take action in response to a food reward, did correlate with the response to calories in the brain reward system. Thus, the response to oral calories is associated with BAS drive, independent of stimulus valence.

Brain responses during food-viewing have been found to correlate with reward sensitivity in the caudate, amygdala and ACC (Beaver et al., 2006; Schienle et al., 2009). We focused on brain responses during exposure to another food-cue: the presence of calories in the oral cavity. In line with the foodviewing studies, we found that taste activation in the striatum, amygdala and ACC is correlated with reward sensitivity. Both the striatum and ACC are important in encoding food reward. They were found to be consistently activated in a meta-analysis of 28 studies in response to a pleasant tastant (Sescousse et al., 2013). The amygdala has also been implicated in food reward (Smeets et al., 2006; Grabenhorst et al., 2010; Jacobson et al., 2010) In addition, several other studies found that the striatum, ACC and amygdala are also involved in the neural encoding of oral calories (Frank et al., 2008; Smeets et al., 2011; Griffioen-Roose et al., 2013) Our results extend this by showing that activation in response to oral calories in the ACC, caudate and amygdala varies with the degree to which individuals are sensitive to reward.

We found an inverse relationship between reward sensitivity and the brain response to oral calories in the amygdala, ACC and caudate. The amygdala plays a central role in the emotional processing of sensory stimuli (Zald, 2003; Costafreda et al., 2008; Sergerie et al., 2008). Aversive stimuli have been found to activate the amygdala (Zald et al., 1998; O’Doherty et al., 2001). However, positive stimuli may also deactivate it (Zald, 2003). This might explain our inverse relationship in the amygdala, because calories can been seen as positive stimuli. In line with this, previous research also showed that tasting a caloric soft drink deactivates the amygdala (Smeets et al., 2011).

Concerning the caudate, Smeets et al. (2011) showed an opposite effect, namely that tasting a caloric soft drink resulted in more activation than tasting a non-caloric one. Few studies with a fMRI-taste paradigm have reported deactivation in the striatum. At this moment, it is known that omission of an expected reward can produce deactivations in the ventral striatum (McClure et al., 2003; O'Doherty, 2004). However, in the current study there was no negative prediction error, thus this cannot explain the negative correlation in the striatum. We speculate that an alternative explanation for caudate deactivation might be the firing of GABA-neurons. The basal ganglia exert inhibitory control over several motor areas via GABAergic output (Hikosaka, 2007). The presence of calories in the mouth compared to a non-caloric liquid, might induce such firing to inhibit motor movements such as searching for other foods. GABA-neurons of individuals with a higher reward-sensitivity level might respond stronger to calories.

Another explanation for the deactivation in the striatum, ACC and amygdala might be that the response to calories in individuals with lower sensitivity to reward is more adapted to internal hunger. If so, they may experience calories as more rewarding during hunger, when calories are necessary for survival, and as less rewarding during satiety, when calories are not necessary. In line with this explanation, we found a positive correlation between reward sensitivity and caudate activation in response to oral calories during satiety. 


\section{HUNGER}

Left ACC

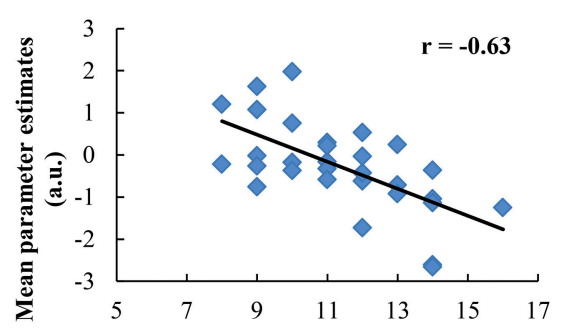

Left ACC

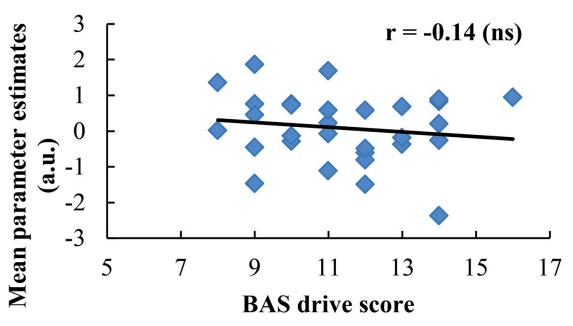

Left: Main peak MNI (0 23 22)

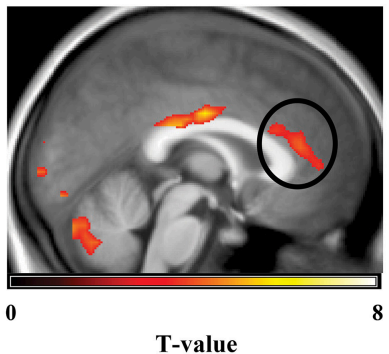

Right ACC

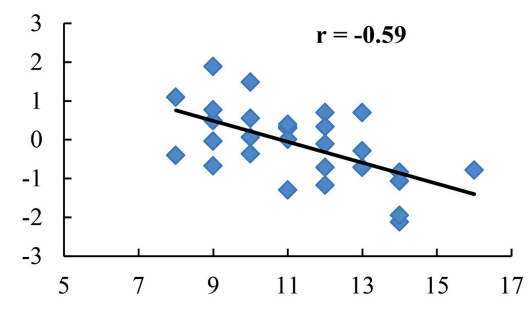

Right ACC

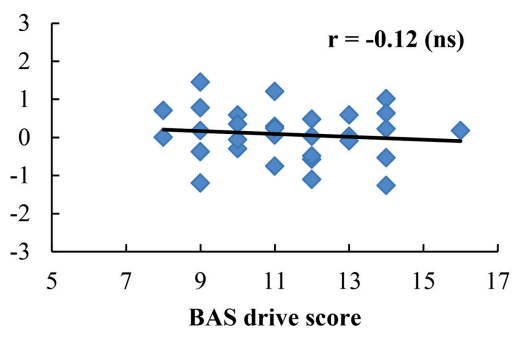

Right: Main peak MNI (3 32 16)

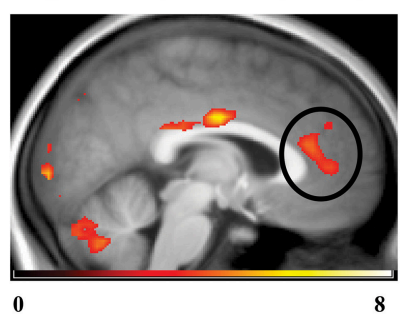

T-value

FIGURE 3 | Scatterplot of brain activation in response to oral calories (maltodextrin and sucralose minus sucralose) and reward sensitivity (BAS drive score) during hunger (significant) and satiety (not significant) in the left and right ACC.

For soft drinks, we found no correlations with reward sensitivity in any reward related area during hunger. The discrepancy between this finding and the associations found for simple solutions can be explained in a number of ways. Firstly, the source of calories was different: maltodextrin vs. sucrose. Sucrose activates the sweet taste receptor, i.e., calories from sucrose are signaled by sweetness. On the contrary, maltodextrin, a tasteless substance for humans (Sclafani and Mann, 1987), is most likely directly detected by an oral maltodextrin receptor, independent of sweet taste (Lapis et al., 2014). In line with this, brain activation is different for a simple sugar compared to maltodextrin (Chambers et al., 2009). Thus, the different calorie sources may trigger different signaling mechanisms, which could have led to different results. In addition, it must be noted that we did not explicitly test for and excluded maltodextrin-tasters in the study with simple solutions. Previous research showed that a small percentage of the population can taste maltodextrin (de Araujo et al., 2013). This could have amplified the results. Secondly, one study used unfamiliar solutions whereas the other study used familiar products (soft drinks that were very similar to commercially available variants). In both studies, we only included participants that consumed more sugar sweetened than artificially sweetened beverages in daily life. Therefore, we assume that participants were conditioned to link the flavor of the soft drinks to calories (Appleton et al., 2006; Brunstrom and Mitchell, 2007; O'Sullivan et al., 2010). This conditioning might have overruled the effect of actual caloric content. Thirdly, the studies used participants of different genders. This may have led to dissimilar results because male and female brain responses to food can differ (Smeets et al., 2006; Uher et al., 2006; Cornier et al., 2010; Frank et al., 2010; Haase et al., 2011). In particular, women may respond stronger to external food-related stimuli than men (Uher et al., 2006; Frank et al., 2010), which could explain why we find effects for women, but not men. Finally, the study with mainly men included fewer participants $(n=$ 18). Many fMRI papers with a tasting-paradigm have used a comparable sample size and have shown significant results, for example: Bender et al. (2009) $(n=19)$, Frank et al. (2008) $(n=$ 

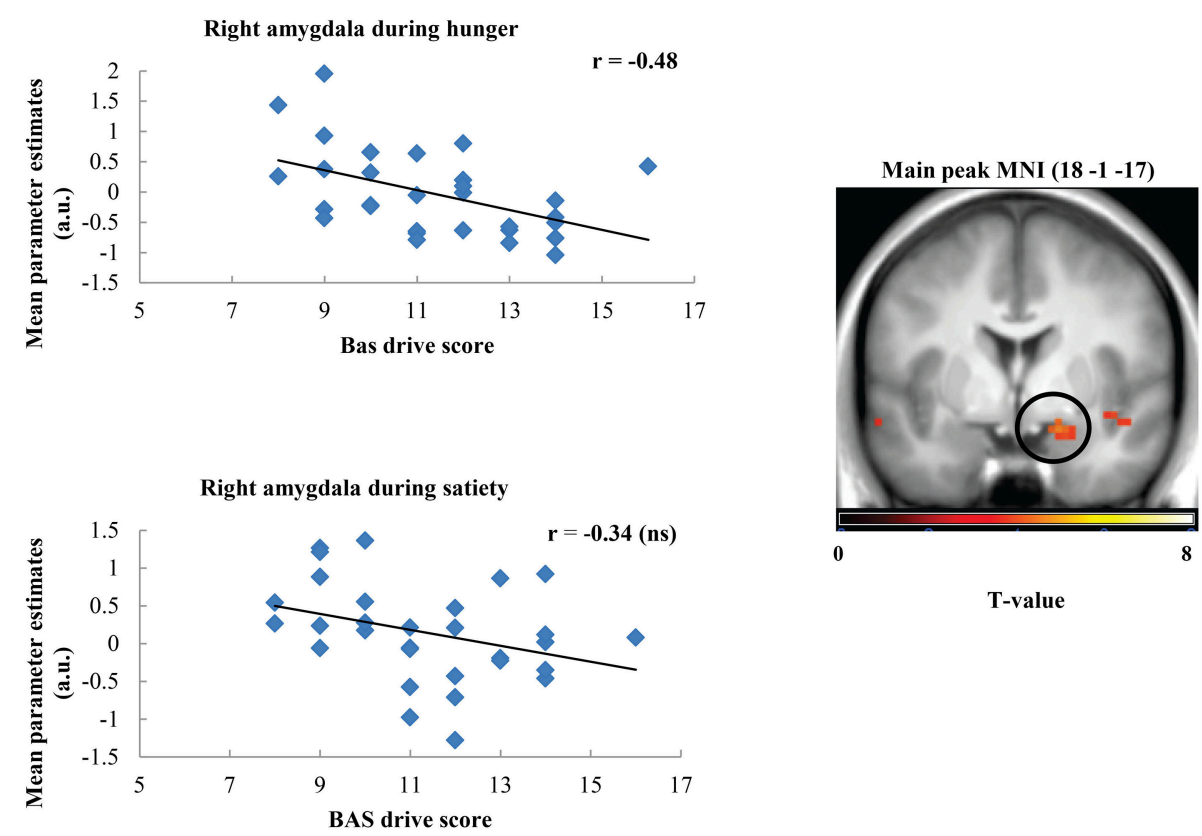

T-value

FIGURE 4 | Scatterplot of brain activation in response to oral calories (maltodextrin and sucralose minus sucralose) and reward sensitivity (BAS drive score) during hunger (significant) and satiety (not significant) in the right amygdala.

12), Haase et al. (2009) ( $n=18)$, O’Doherty et al. (2001) $(n=7)$, Spetter et al. (2010) $(n=15)$. Nevertheless, it is possible that the relatively low sample size has prevented detection of small effects.

\section{CONCLUSION}

We found that neural responses to oral calories from a maltodextrin solution are modulated by reward sensitivity in reward-related areas such as the caudate, amygdala, and ACC. This was not the case for a sucrose sweetened soft drink. This discrepancy may be due to the direct detection of maltodextrin, but not sucrose in the oral cavity. Also, in a familiar drink (soft drink), detection of calories per se may be overruled by a conditioned response to the familiar flavor. In conclusion, the brain reward response to calories from a long chain starch sugar (maltodextrin) varies with reward sensitivity. The absence of this effect in a familiar soft drink warrants further research into its relevance for real life ingestive behavior.

\section{AUTHOR CONTRIBUTIONS}

IV, SG, CD, PS: Substantial contributions to the conception or design of the work; or the acquisition, analysis, or interpretation of data for the work; IV, SG, CD, PS: Drafting the work or revising it critically for important intellectual content; IV, SG, CD, PS: Final approval of the version to be published; IV, SG, CD, PS: Agreement to be accountable for all aspects of the work in ensuring that questions related to the accuracy or integrity of any part of the work are appropriately investigated and resolved.

\section{FUNDING}

This work is part of the FOCOM project which was supported by the European Regional Development Fund and the Dutch Provinces Gelderland and Overijssel (Grant number 2011017004).

\section{ACKNOWLEDGMENTS}

We would like to express our thanks to all partners of the FOCOM project for their valuable input. Furthermore, the use of the 3T MRI facility has been made possible by CAT-AgroFood (Shared Research Facilities Wageningen UR).

\section{SUPPLEMENTARY MATERIAL}

The Supplementary Material for this article can be found online at: http://journal.frontiersin.org/article/10.3389/fnbeh. 2015.00371 


\section{REFERENCES}

Appleton, K. M., Gentry, R. C., and Shepherd, R. (2006). Evidence of a role for conditioning in the development of liking for flavours in humans in everyday life. Physiol. Behav. 87, 478-486. doi: 10.1016/j.physbeh.2005. 11.017

Beaver, J. D., Lawrence, A. D., van Ditzhuijzen, J., Davis, M. H., Woods, A., and Calder, A. J. (2006). Individual differences in reward drive predict neural responses to images of food. J. Neurosci. 26, 5160-5166. doi: 10.1523/JNEUROSCI.0350-06.2006

Bender, G., Veldhuizen, M. G., Meltzer, J. A., Gitelman, D. R., and Small, D. M. (2009). Neural correlates of evaluative compared with passive tasting. Eur. J. Neurosci. 30, 327-338. doi: 10.1111/j.1460-9568.2009.06819.x

Bijttebier, P., Beck, I., Claes, L., and Vandereycken, W. (2009). Gray' $s$ reinforcement sensitivity theory as a framework for research on personality-psychopathology associations Clin. Psychol. Rev. 29, 421-430. doi: 10.1016/j.cpr.2009.04.002

Brunstrom, J. M., and Mitchell, G. L. (2007). Flavor-nutrient learning in restrained and unrestrained eaters. Physiol. Behav. 90, 133-141. doi: 10.1016/j.physbeh.2006.09.016

Carver, C. S., and White, T. L. (1994). Behavioral inhibition, behavioral activation and affective responses to impending reward and punishment: the BIS/BAS Scales. J. Pers. Soc. Psychol. 67, 319-333.

Chambers, E. S., Bridge, M. W., and Jones, D. A. (2009). Carbohydrate sensing in the human mouth: effects on exercise performance and brain activity. J. Physiol. 587, 1779-1794. doi: 10.1113/jphysiol.2008.164285

Cornier, M. A., Salzberg, A. K., Endly, D. C., Bessesen, D. H., and Tregellas, J. R. (2010). Sex-based differences in the behavioral and neuronal responses to food. Physiol. Behav. 99, 538-543. doi: 10.1016/j.physbeh.2010.01.008

Corr, P. J., Deyoung, C. G., and Mcnaughton, N. (2013). Motivation and personality: a neuropsychological perspective. Soc. Pers. Psychol. Compass 7, 158-175. doi: $10.1111 /$ spc3.12016

Costafreda, S. G., Brammer, M. J., David, A. S., and Fu, C. H. Y. (2008). Predictors of amygdala activation during the processing of emotional stimuli: a meta-analysis of 385 PET and fMRI studies. Brain Res. Rev. 58, 57-70. doi: 10.1016/j.brainresrev.2007.10.012

Davis, C., Patte, K., Levitan, R., Reid, C., Tweed, S., and Curtis, C. (2007). From motivation to behaviour: a model of reward sensitivity, overeating, and food preferences in the risk profile for obesity. Appetite 48, 12-19. doi: 10.1016/j.appet.2006.05.016

Davis, C., Strachan, S., and Berkson, M. (2004). Sensitivity to reward: implications for overeating and overweight. Appetite 42, 131-138. doi: 10.1016/j.appet.2003.07.004

de Araujo, I. E., Lin, T., Veldhuizen, M. G., and Small, D. M. (2013). Metabolic regulation of brain response to food cues. Curr. Biol. 23, 878-883. doi: 10.1016/j.cub.2013.04.001

Frank, G. K. W., Oberndorfer, T. A., Simmons, A. N., Paulus, M. P., Fudge, J. L., Yang, T. T., et al. (2008). Sucrose activates human taste pathways differently from artificial sweetener. Neuroimage 39, 1559-1569. doi: 10.1016/j.neuroimage.2007.10.061

Frank, S., Laharnar, N., Kullmann, S., Veit, R., Canova, C., Hegner, Y. L., et al. (2010). Processing of food pictures: influence of hunger, gender and calorie content. Brain Res. 1350, 159-166. doi: 10.1016/j.brainres.2010.04.030

Franken, I. H. A., and Muris, P. (2005). Individual differences in reward sensitivity are related to food craving and relative body weight in healthy women. Appetite 45, 198-201. doi: 10.1016/j.appet.2005.04.004

Franken, I. H. A., Muris, P., and Rassin, E. (2005). Psychometric properties of the Dutch BIS/BAS Scales. J. Psychopathol. Behav. Assess. 27, 25-30. doi: 10.1007/s10862-005-3262-2

Gomez, R., Cooper, A., and Gomez, A. (2005)., An item response theory analysis of the Carver and White (1994). BIS/BAS Scales. Pers. Individ. Dif. 39, 1093-1103. doi: $10.1016 /$ j.paid.2005.03.015

Grabenhorst, F., Rolls, E. T., Parris, B. A., and d'Souza, A. A. (2010). How the brain represents the reward value of fat in the mouth. Cereb. Cortex 20, 1082-1091. doi: 10.1093/cercor/bhp169

Gray, J. A. (1990). Brain systems that mediate both emotion and cognition. Cogn. Emot. 4, 269-288. doi: 10.1080/02699939008410799
Gray, J. A., and Mcnaughton, N. (2003). The Neuropsychology of Anxiety: An Enquiry into the Functions of the Septo-Hippocampal System, 2nd Edn. Oxford: Oxford University Press.

Griffioen-Roose, S., Smeets, P. A. M., Weijzen, P. L. G., van Rijn, I., and van den Bosch, I., de Graaf, C. (2013). Effect of replacing sugar with non-caloric sweeteners in beverages on the reward value after repeated exposure. PLoS ONE 8:e81924. doi: 10.1371/journal.pone.0081924

Haase, L., Cerf-Ducastel, B., and Murphy, C. (2009). Cortical activation in response to pure taste stimuli during the physiological states of hunger and satiety. Neuroimage 44, 1008-1021. doi: 10.1016/j.neuroimage.2008.09.044

Haase, L., Green, E., and Murphy, C. (2011). Males and females show differential brain activation to taste when hungry and sated in gustatory and reward areas. Appetite 57, 421-434. doi: 10.1016/j.appet.2011.06.009

Harrison, A., O’Brien, N., Lopez, C., and Treasure, J. (2010). Sensitivity to reward and punishment in eating disorders. Psychiatry Res. 177, 1-11. doi: 10.1016/j.psychres.2009.06.010

Hikosaka, O. (2007). GABAergic output of the basal ganglia. Prog. Brain Res. 160, 209-226. doi: 10.1016/S0079-6123(06)60012-5

Jacobson, A., Green, E., and Murphy, C. (2010). Age-related functional changes in gustatory and reward processing regions: an fMRI study. Neuroimage 53, 602-610. doi: 10.1016/j.neuroimage.2010.05.012

Lapis, T. J., Penner, M. H., and Lim, J. (2014). Evidence that humans can taste glucose polymers. Chem. Senses 39, 737-747. doi: 10.1093/chemse/bju031

Maldjian, J. A., Laurienti, P. J., Kraft, R. A., and Burdette, J. H. (2003). An automated method for neuroanatomic and cytoarchitectonic atlasbased interrogation of fMRI data sets. Neuroimage 19, 1233-1239. doi: 10.1016/S1053-8119(03)00169-1

McClure, S. M., Berns, G. S., and Montague, P. R. (2003). Temporal predection errors in a passive learning task activate human striatum. Neuron 38, 339-346. doi: 10.1016/S0896-6273(03)00154-5

Ng, M., Fleming, T., Robinson, M., Thomson, B., Graetz, N., Margono, C., et al. (2014). Global, regional, and national prevalence of overweight and obesity in children and adults during 1980-2013: a systematic analysis for the global burden of disease study 2013. Lancet 384, 766-781. doi: 10.1016/S01406736(14)60460-8

O'Doherty, J. P. (2004). Reward representations and reward-related learning in the human brain: insights from neuroimaging. Curr. Opin. Neurobiol. 14, 769-776. doi: 10.1016/j.conb.2004.10.016

O'Doherty, J., Rolls, E. T., Francis, S., Bowtell, R., and McGlone, F. (2001). Representation of pleasant and aversive taste in the human brain. J. Neurophysiol. 85, 1315-1321.

O’Sullivan, H. L., Alexander, E., Ferriday, D., and Brunstrom, J. M. (2010). Effects of repeated exposure on liking for a reduced-energy-dense food. Am. J. Clin. Nutr. 91, 1584-1589. doi: 10.3945/ajcn.2009.28863

Schienle, A., Schäfer, A., Hermann, A., and Vaitl, D. (2009). Binge-eating disorder: reward sensitivity and brain activation to images of food. Biol. Psychiatry 65, 654-661. doi: 10.1016/j.biopsych.2008.09.028

Sclafani, A., and Mann, S. (1987). Carbohydrate taste preferences in rats: glucose, sucrose, maltose, fructose and polycose compared. Physiol. Behav. 40, 563-568.

Sergerie, K., Chochol, C., and Armony, J. L. (2008). The role of the amygdala in emotional processing: a quantitative meta-analysis of functional neuroimaging studies. Neurosci. Biobehav. Rev. 32, 811-830. doi: 10.1016/j.neubiorev.2007.12.002

Sescousse, G., Caldú, X., Segura, B., and Dreher, J.-C. (2013). Processing of primary and secondary rewards: a quantitative meta-analysis and review of human functional neuroimaging studies. Neurosci. Biobehav. Rev. 37, 681-696. doi: 10.1016/j.neubiorev.2013.02.002

Smeets, P. A. M., de Graaf, C., Stafleu, A., van Osch, M. J. P., Nievelstein, R. A. J., and van der Grond, J. (2006). Effect of satiety on brain activation during chocolate tasting in men and women. Am. J. Clin. Nutr. 83, 1297-1305.

Smeets, P. A. M., Weijzen, P., de Graaf, C., and Viergever, M. A. (2011). Consumption of caloric and non-caloric versions of a soft drink differentially affects brain activation during tasting. Neuroimage 54, 1367-1374. doi: 10.1016/j.neuroimage.2010.08.054

Spetter, M. S., Smeets, P. A. M., de Graaf, C., and Viergever, M. A. (2010). Representation of sweet and salty taste intensity in the brain. Chem. Senses 35, 831-840. doi: 10.1093/chemse/bjq093 
Uher, R., Treasure, J., Heining, M., Brammer, M. J., and Campbell, I. C. (2006). Cerebral processing of food-related stimuli: effects of fasting and gender. Behav. Brain Res. 169, 111-119. doi: 10.1016/j.bbr.2005.12.008

van Rijn, I., de Graaf, C., and Smeets, P. A. M. (2015). Tasting calories differentially affects brain activation during hunger and satiety. Behav. Brain Res. 279, 139-147. doi: 10.1016/j.bbr.2014.11.019

van Strien, T. (2005). Dutch Eating Behavior Questionnaire Manual [Handleiding Voor de Nederlandse Vragenlijst Voor Eetgedrag], 1st Edn. Amsterdam: Boom Test Publishers.

Verbeken, S., Braet, C., Lammertyn, J., Goossens, L., and Moens, E. (2012). How is reward sensitivity related to bodyweight in children? Appetite 58, 478-483. doi: 10.1016/j.appet.2011.11.018

Zald, D. H. (2003). The human amygdala and the emotional evaluation of sensory stimuli. Brain Res. Rev. 41, 88-123. doi: 10.1016/S0165-0173(02)00248-5
Zald, D. H., Lee, J. T., Fluegel, K. W., and Pardo, J. V. (1998). Aversive gustatory stimulation activates limbic circuits in humans. Brain 121 (Pt 6), 1143-1154.

Conflict of Interest Statement: The authors declare that the research was conducted in the absence of any commercial or financial relationships that could be construed as a potential conflict of interest.

Copyright $\odot 2016$ van Rijn, Griffioen-Roose, de Graaf and Smeets. This is an openaccess article distributed under the terms of the Creative Commons Attribution License (CC BY). The use, distribution or reproduction in other forums is permitted, provided the original author(s) or licensor are credited and that the original publication in this journal is cited, in accordance with accepted academic practice. No use, distribution or reproduction is permitted which does not comply with these terms. 